\title{
Investigation of the Non-Square InGaAsP/InP Quantum Wells in the Electric Field by Photoreflectance
}

R. Kudrawiec* , G. Sęk, W. Rudno-Rudiński, J. Misiewicz

Institute of Physics, Wroctaw University of Technology

Wybrzeże Wyspiańskiego 27, 50-370 Wrocław, Poland

J. Wojcik, B.J. Robinson, D.A. Thompson and P. Mascher

Centre for Electrophotonic Materials and Devices

Department of Engineering Physics

McMaster University, Hamilton, Ontario, L8S 4L7 Canada

\begin{abstract}
Non-square quantum wells in electric field have been investigated by photoreflectance and photoluminescence spectroscopies. The structures have been obtained by a post-growth modification (rapid thermal annealing) of standard $1.55 \mu \mathrm{m}$ InGaAsP-based laser structures that were grown by gas source molecular beam epitaxy. During rapid thermal annealing a migration of semiconductor atoms across quantum well interfaces changes the quantum well profile from a square well to a rounded well. The modification of the profile changes energy levels in the quantum well and in consequence a blue shift of the quantum well emission peak is observed in photoluminescence. In this paper the blue shift of the ground state transition of post-growth modified quantum well structures has been investigated by both photoluminescence and photoreflectance techniques. Also a blue shift of excited state transitions has been observed in photoreflectance spectra. Generally, a stronger blue shift for the ground state transition than for excited state transitions has been observed. Additionally, oscillator strengths for all quantum well transitions have been determined from photoreflectance spectra. It has been found that the oscillator strength is constant for all quantum well transitions despite of modification of the quantum well profile.
\end{abstract}

PACS numbers: 73.63.Hs, 78.67.-n, 66.10.Cb

*corresponding author; e-mail: kudrawie@if.pwr.wroc.pl 


\section{Introduction}

Over the past three decades there has been a rapid and intensive development of quantum well (QW) structures, and their application in optical devices. However, this development has been based predominantly on square QWs (i.e., sharp, step-like interfaces between well and barrier material). Non-square QWs have been investigated less intensively. A recent increase in interest in QW structures operating in the $1.3-1.5 \mu \mathrm{m}$ spectral region has caused an increase in applications of the post-growth techniques [1] that produce non-square QWs, for a modification of the operation wavelength of well-known InP-based laser structures [2,3]. So far many post-growth techniques including ion implantation, laser heating, and rapid thermal annealing (RTA) [1] have been applied to induce a band gap modification of QW structures. Generally, in all cases a migration of semiconductor atoms between QW interfaces has been observed. The interdiffusion of atoms across the heterointerface in an as-grown square QW causes a modification of the composition and confinement profiles of the QW. The modification influences energy levels of the QW. This effect in literature is often called quantum well intermixing (QWI) [1]. Optical properties of such modified QWs are mostly investigated by photoluminescence (PL) (emission-like experiment). Absorption-like experiments, which make possible to study the excited states, have been rarely used. This work presents an application of the photoreflectance technique, which is an absorption-like method, to investigate both ground and excited states QW transitions of the post-growth modified laser structures. Photoreflectance (PR), according to our knowledge, has been applied for the first time in such InGaAsP-based QW structures. Samples used for this study have been capped by $\mathrm{SiO}_{2}$ film and processed by rapid thermal annealing, which produces vacancies at the $\mathrm{SiO}_{2}$ /semiconductor interface. The vacancies diffuse rapidly down to the QW region enhancing the compositional intermixing [3].

For the non-modified laser structure interband transitions from heavy- and light-hole in valence subbands to conduction subbands have been observed and identified in the PR spectra. The values of the transition energies obtained from the experiment agree with the values obtained from the theoretical calculations using an envelope function model. We investigate the evolution of the QW-related optical transitions versus the conditions of the post-growth processes.

\section{Experiment}

Samples used in this study were grown by gas source molecular beam epitaxy (GSMBE) on $n$-doped (100) InP substrates. The laser structure is shown schematically in Fig. 1, where labels $1.15 \mathrm{Q}$ and $1.24 \mathrm{Q}$ mean quaternary layers with energy gap equivalent to 1.15 and $1.24 \mu \mathrm{m}$, respectively. The active region is composed of three compressively strained $5 \mathrm{~nm}$ thick QWs of $\operatorname{In}_{0.76} \mathrm{Ga}_{0.24} \mathrm{As}_{0.85} \mathrm{P}_{0.15}$ separated by $10 \mathrm{~nm}$ barriers of $1.24 \mathrm{Q}\left(\mathrm{In}_{0.76} \mathrm{Ga}_{0.24} \mathrm{As}_{0.52} \mathrm{P}_{0.48}\right)$ lattice-matched to 


\begin{tabular}{|c|}
\hline $\operatorname{InP}-$ Shicld $(500 \AA$ ud $)$ \\
\hline $\mathrm{In}_{0.53} \mathrm{Ga}_{0.47} \operatorname{As}(1000 \AA ;$ ud $)$ \\
\hline $\operatorname{InP}(250 \AA \mathrm{u}$ ud $)$ \\
\hline 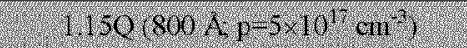 \\
\hline $1.24 \mathrm{Q}(700 \AA ; \mathrm{ud})$ \\
\hline $3 \times 0 W(50 \wedge$ well, 100 A barier, ud) \\
\hline $1.24 \mathrm{Q}(700 \AA ; \mathrm{ud})$ \\
\hline 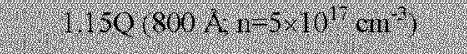 \\
\hline $\operatorname{InP}\left(5000 \AA ; \mathrm{n}=1 \times 10^{18} \mathrm{~cm}^{-3}\right)$ \\
\hline Substrate n-type InP \\
\hline $\begin{array}{l}1.15 \mathrm{Q} \rightarrow \mathrm{In}_{0.76} \mathrm{Ga}_{0.24} \mathrm{As}_{0.39} \mathrm{P}_{0.61} \\
1.24 \mathrm{Q} \rightarrow \mathrm{In}_{0.76} \mathrm{Ga}_{0.24} \mathrm{As}_{0.85} \mathrm{P}_{0.15}\end{array}$ \\
\hline
\end{tabular}

Fig. 1. Standard $1.55 \mu \mathrm{m}$ InGaAsP-based 3QW laser structure.

InP substrate. This gives an emission at $1568 \mathrm{~nm}$ wavelength as determined by room temperature photoluminescence. The content has been chosen so that the In/Ga ratio remains constant throughout the wells and barriers. This "partial" laser structure is completed with $80 \mathrm{~nm}$ upper and lower cladding regions of a 1.15 quaternary $\left(\mathrm{In}_{0.76} \mathrm{Ga}_{0.24} \mathrm{As}_{0.39} \mathrm{P}_{0.61}\right)$ layer doped with $5 \times 10^{17} \mathrm{~cm}^{-3}$ Be and $\mathrm{Si}$, respectively and capped with undoped InP, InGaAs, and InP layers, as shown in Fig. 1. The InP shield had been removed before the deposition of the $\mathrm{SiO}_{2}$ film. Silicon dioxide was deposited by electron cyclotron resonance plasma enhanced chemical vapour deposition (ECR-PECVD). The technical details of this system have been described elsewhere [4]. The samples have been obtained at various microwave powers of ECR-PECVD process and the same conditions of RTA ( 60 seconds and $750^{\circ} \mathrm{C}$ ). The microwave power was changed from 600 to $800 \mathrm{~W}$. For optical characterisation the dielectric film and next two layers (InGaAs, InP) had been removed.

The PR measurements were performed in the so-called bright configuration where the sample is illuminated by white light from a halogen lamp (100 W) serving as a probe beam source, at near normal incidence. The reflected light was dispersed through a $0.55 \mathrm{~m}$ focal length single grating monochromator and detected by a germanium photodiode. For photomodulation $488 \mathrm{~nm}$ line of an $\mathrm{Ar}^{+}$ laser was used as a pump beam. 


\section{Results and discussion}

Figure 2 shows PR spectra recorded for as-grown (\#1), as-grown annealed $(\# 2)$, and $\mathrm{SiO}_{2}$ film capped annealed samples $(\# 3-\# 7)$. For samples labelled as $\# 3$, \#4, \#5, \#6, and \#7 the microwave power is $600,650,700,750,800 \mathrm{~W}$, respectively. Open circles represent experimental points, solid lines represent fits with PR theoretical curves, most appropriate for confined state transitions at room temperature [5]. In order to assign the features seen in Fig. 2, the Schrödinger

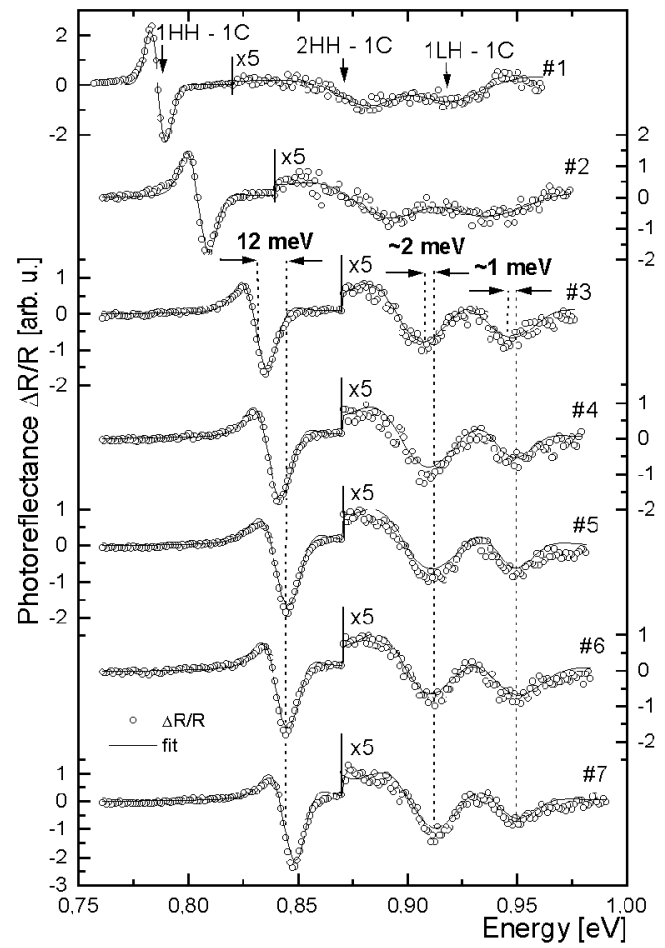

Fig. 2. Room temperature PR spectra of the laser structures (\#1 - as-grown, \#2 as-grown annealed, \#3-, ., \#7- $\mathrm{SiO}_{2}$ film capped annealed samples). Open circles - experimental points, solid lines — fitting curves.

equation has been solved for the structure of the as-grown sample in the envelope function approximation. The energy levels for different transition were obtained assuming a single square quantum well, tilted by a $p-i-n$ junction built-in field of $57 \mathrm{kV} / \mathrm{cm}$. This field is estimated assuming the pining of the Fermi level at acceptor and donor levels in the surrounding quantum well layers. This way the origin of the observed three PR resonances has been explained. The first resonance at $0.790 \mathrm{eV}$ is associated with transition between the first heavy hole subband and the first electron subband (1HH-1C). In PL spectrum the emission peak has been 
observed at the same energy. The second resonance at $0.888 \mathrm{eV}$ (labelled $2 \mathrm{HH}-1 \mathrm{C}$ ) is associated with transition between the second heavy hole subband and the first electron subband. Generally, the $2 \mathrm{HH}-1 \mathrm{C}$ transition is forbidden, but in this case the electric field of the junction breaks the selection rules and the transition may be observed. The third resonance at $0.910 \mathrm{eV}(1 \mathrm{LH}-1 \mathrm{C})$ is associated with transition between the first light hole subband and the first electron subband. The post-growth modification of the QW shifts the energy levels in the well, but the order of PR features remains the same. In PR spectra of samples \#2 and \#3 (Fig. 2) different values of the blue shift are observed for all three transitions. The strongest shift is observed in the ground state transition (1HH-1C) and it reaches $16 \mathrm{meV}$ and $44 \mathrm{meV}$ for sample \#2 and sample \#3, respectively. The other two higher energy transitions (2HH-1C and $1 \mathrm{LH}-1 \mathrm{C}$ ) are clearly less shifted. A very small blue shift is observed for $2 \mathrm{HH}-1 \mathrm{C}$ transition. It is only $2 \mathrm{meV}$ and $11 \mathrm{meV}$ for sample \#2 and sample \#3, respectively, whereas for 1LH-1C transition the blue shift equals $11 \mathrm{meV}$ and $34 \mathrm{meV}$, respectively. The comparison of samples \#2 and \#3 shows that the existence of the dielectric film is conductive to the atom migration across QW interfaces. The dielectric cap induced enhancement of the blue shift is 38,10 , and $24 \mathrm{meV}$ for $1 \mathrm{HH}-1 \mathrm{C}, 2 \mathrm{HH}-1 \mathrm{C}$, and $1 \mathrm{LH}-1 \mathrm{C}$ transitions, respectively. Also the microwave power of the ECR-PECVD process plays a significant role. It has been shown that the increase in the power from 600 to $800 \mathrm{~W}$ generates an increase in the blue shift of the ground state transition from 44 to $56 \mathrm{meV}$, while the energy of the next two (2HH-1C, $1 \mathrm{LH}-1 \mathrm{C})$ transitions changes less than $2 \mathrm{meV}$ (see in Fig. 3). Such a small shift of excited state transitions means that the change of the QW profile does not influence the total energy $E_{\mathrm{t}}$

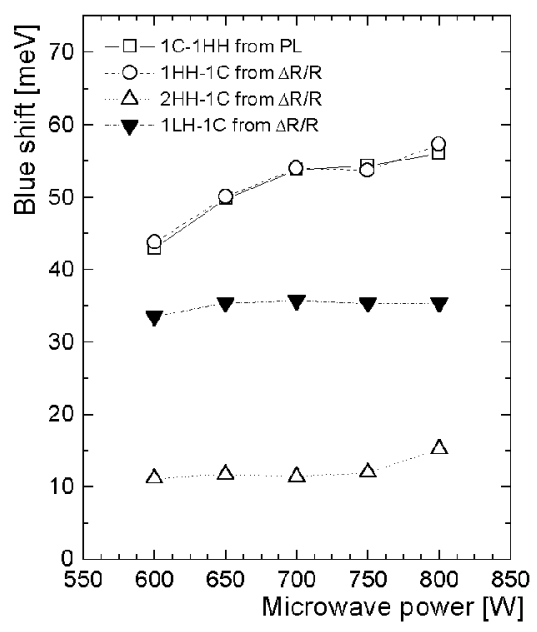

Fig. 3. Dependence of the blue shift on the microwave power of ECR-PECVD process. 
of these transitions, but nevertheless the energies of electron and hole levels could have changed. The quantity $E_{\mathrm{t}}$ is defined as

$$
E_{\mathrm{t}}=E_{\mathrm{g}}+E_{\mathrm{e}}+E_{\mathrm{h}},
$$

where $E_{\mathrm{g}}, E_{\mathrm{e}}$, and $E_{\mathrm{h}}$ are the band gap of the QW material, energy of electron, and hole levels, respectively. It is expected that the electron $E_{\mathrm{e}}$ and hole $E_{\mathrm{h}}$ energies change as a result of modification of the QW profile. It can be indirectly confirmed in PL experiment (basing on the intensity changes). Figure 4 shows PL spectra for samples \#3 - \#7. The inset shows integrated PL intensity of the ground state transition versus its blue shift. A decrease in intensity is observed with the increase in the shift. It can be explained by a reduction of the energy distance between energy level and the top of QW potential, i.e. the easier thermal escape of the carriers from the well.

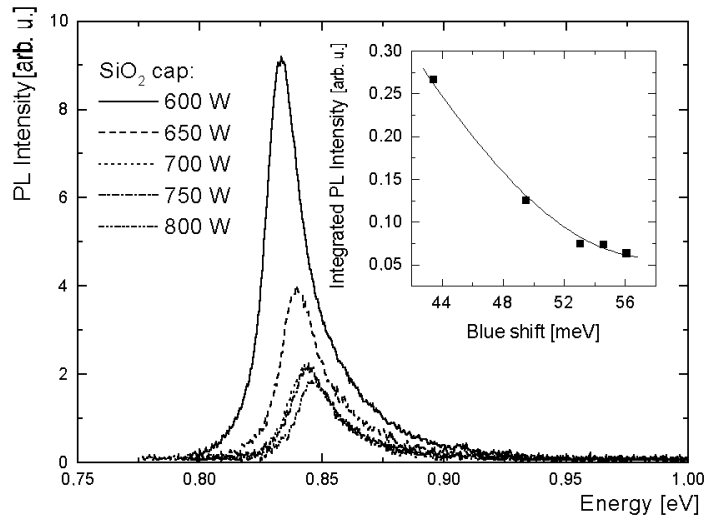

Fig. 4. Room temperature PL spectra for $\mathrm{SiO}_{2}$ capped samples. The inset shows integrated intensity of $\mathrm{PL}$ peak versus its blue shift.

The post-growth structure modification changes the profile of the well so that the well becomes shallower and the levels shift to the edge of the potential barrier. All the features seen in Fig. 2 are related to transitions between the first electron subband and three different hole subbands: $1 \mathrm{HH}, 2 \mathrm{HH}$, and $1 \mathrm{LH}$. The capping with a dielectric film and annealing shifts the levels and the transition energies. Also the changes of microwave power influence the energy levels, mainly for the ground state transition (12 meV shift). The energies of higher transitions are almost unchanged that reflects the magnitude of the hole levels shift. It shows that significantly shifted is only the ground heavy hole level - the one closer to the bottom of the well.

There has not been observed any Stokes shift between PL and PR features of the fundamental transition. The lack of the shift shows that the post-growth modification changes the profile of the well but do not alter the quality of the layers. 
The magnitude of the oscillator strength of QW transitions versus the blue shift of the ground state transition has been investigated using Kramers-Krönig analysis (KKA) $[6,7]$. The modulus of PR signal of samples \#1, \#2, and \#6 is shown in Fig. 5. The integrated field of the obtained peaks can be interpreted as the oscillator strength in arbitrary units. Generally, it has been obtained that the oscillator strength of these three QW transitions does not change within experimental error (see Fig. 6). The intensity (amplitude) of the 1HH-1C transition

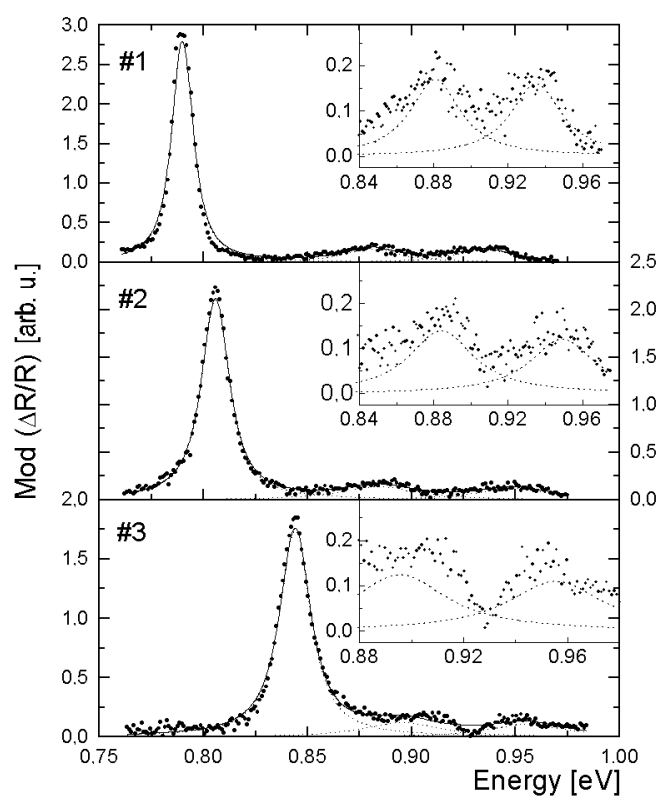

Fig. 5. Modulus of the PR spectrum of as-grown \#1, as-grown annealed \#2, and $8 \mathrm{SiO}_{2}$ capped annealed \#6 samples. Insets show the modulus of PR spectrum in shorter spectral range.

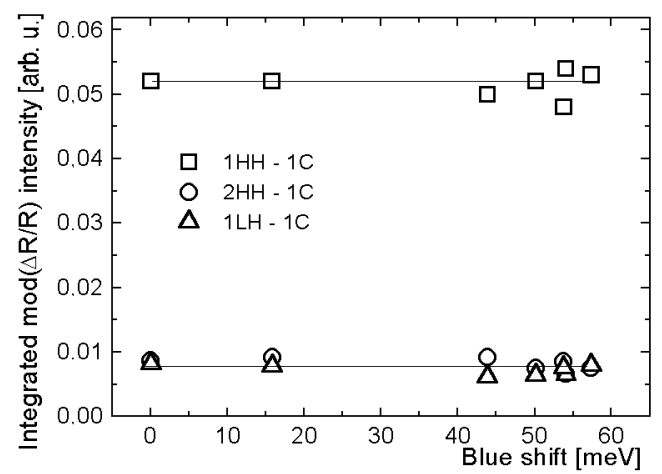

Fig. 6. Oscillator strengths versus the blue shift of the ground state transition. 


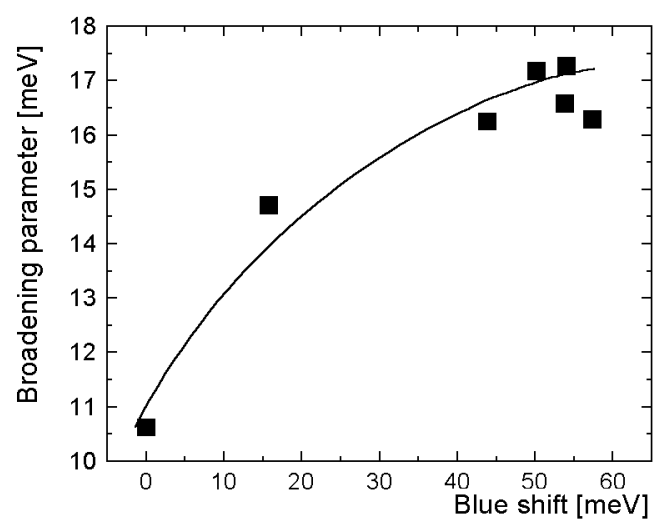

Fig. 7. The broadening parameter of the ground state transition versus its blue shift.

decreases with an increase in its energy, but the broadening parameter of this transition increases such that the integrated intensity of the $1 \mathrm{HH}-1 \mathrm{C}$ peak is constant (the value of the $1 \mathrm{HH}-1 \mathrm{C}$ broadening parameter versus the $1 \mathrm{HH}-1 \mathrm{C}$ blue shift is shown in Fig. 7). The same value of oscillator strength means that the symmetry of wave functions for electron and holes does not change significantly after the QW modification.

\section{Conclusion}

In summary, non-square quantum wells in built-in electric field have been investigated by photoreflectance and photoluminescence spectroscopy. For post-growth modified QWs structures the blue shift has been observed for both ground and excited state transitions. It has been found that the ground state transition is mostly sensitive to the band gap modification. Significant enhancement of the blue shift has been observed for structures capped with $\mathrm{SiO}_{2}$ film before rapid thermal annealing. Also, it has been found that the microwave power of ECR-PECVD process plays a significant role in the band gap modification. Similarly, as time and/or temperature of RTA process, the microwave power may be a good parameter for the control of the blue shift and hence post-growth correction of the laser emission wavelength. Additionally, from PR spectra the oscillator strength for all QW transitions has been derived. It has been observed that in spite of modification of the QW profile the oscillator strength is constant for all transitions.

\section{Acknowledgments}

One of the authors (G.S.) acknowledges the financial support from the Foundation for Polish Science. 


\section{References}

[1] Semiconductor Quantum Well Intermixing, Ed. E.H. Li, Gordon \&Breach, Amsterdam 1997.

[2] J.F. Hazell, D.A. Thompson, N. Bertsch, J.G. Simmons, B.J. Robinson, G.I. Sproule, Semicond. Sci. Technol. 16, 986 (2001).

[3] N. Cao, B.B. Elenkrig, J.G. Simmons, D.A. Thompson, N. Puetz, Appl. Phys. Lett. 70, 3419 (1997).

[4] M. Boudreau, M. Boumerzoug, P. Mascher, P.E. Jessop, Appl. Phys. Lett. 63, 3014 (1993).

[5] F.H. Pollak, Modulation Spectroscopy of Semiconductor and Semiconductor Microstructures, in: Handbook on Semiconductors, Vol. 2, Ed. M. Balkanski, Elsevier Science, Amsterdam 1994, p. 527.

[6] T.J.C. Hosea, Phys. Status Solidi B 189, 531 (1995).

[7] K. Jezierski P. Markiewicz, J. Misiewicz, M. Panek, B. Ściana, R. Korbutowicz, M. Tłaczała, J. Appl. Phys. 77, 4139 (1995). 